\title{
LA TERAPIA DE LA COHERENCIA: UN ENFOQUE CONSTRUCTIVISTA APOYADO POR LA NEUROCIENCIA CONTEMPORÁNEA
}

\section{COHERENCE THERAPY: A CONSTRUCTIVIST APPROACH SUPPORTED BY CONTEMPORARY NEUROSCIENCE}

\author{
Michą JASIŃSKI ${ }^{1}$, ClARA PAZ ${ }^{1}$ Y GUILlEM FEIXAS ${ }^{1}$
}

Cómo referenciar este artículo/How to reference this article:

Jasiński, M., Paz, C., y Feixas, G. (2016). La terapia de la coherencia: un enfoque constructivista apoyado por la neurociencia contemporánea [Coherence therapy: a constructivist approach supported by contemporary neuroscience]. Acción Psicológica, 13(1), 131-144. http://dx.doi.org/10.5944/ap.13.1.16137

\section{Resumen}

En el presente artículo se describe la terapia de la coherencia (TC). Con el fin de presentar este enfoque se realiza una breve descripción del constructivismo y su influencia tanto en la TC, como en la psicoterapia en general. Se continúa con la explicación detallada de la historia, teoría y metodología de la TC. Se describe también la reconsolidación de la memoria, un fenómeno descubierto recientemente en el campo de la neurociencia que parece ocurrir en el proceso de la TC y se presentan sus implicaciones para la psicoterapia. Se utiliza un breve estudio de caso para explicar el proceso de la TC de forma práctica. Finalmente, se describe el estado acual y el futuro de este enfoque terapéutico.
Palabras clave: terapia de la coherencia; constructivismo.

Abstract
Coherence therapy (CT) is described in the present
article. Constructivism's influence on psychotherapy
in general and on CT specifically is discussed briefly,
followed by a detailed presentation of CT's history,
theory and methodology. Memory reconsolidation, a
recently discovered phenomenon which is stated to
occur in CT process, is described as well together
with its implications for psychotherapy field. A brief
case study is presented to show CT's process in prac-
tice and the future and limitations of CT are discussed
in the final part of the article.

Correspondencia: Guilem Feixas. Departamento de Departament de Personalidad, Ealuación y Tratamiento Psicológico, Universidad de Barcelona.

Email: gfeixas@ub.edu

${ }^{1}$ Universidad de Barcelona, España.

Recibido: 07 de marzo de 2016.

Aceptado: 22 de mayo de 2016. 
Keywords: coherence therapy; constructivism.

\section{Introducción}

Para los psicoterapeutas una de las mayores fuentes de satisfacción son las sesiones de terapia en las que de forma inesperada el cliente experimenta un cambio profundo que resulta ser crucial para alcanzar el objetivo terapéutico. El cambio profundo es entendido, de acuerdo con Ecker y Toomey (2008), como una depotenciación de engramas de la memoria implícita que resulta en un cese completo y duradero del síntoma. Experimentar estos cambios produce una mejoría en los síntomas y hace que el individuo se sienta significativamente mejor. Como terapeutas nos gustaría que estas extraordinarias circunstancias se presentasen con mayor frecuencia, pero en la práctica no siempre lo conseguimos.

Bruce Ecker y Laurel Hulley, psicoterapeutas constructivistas inspirados tanto por Bateson (1951, 1972) y Mahoney (1991), pero también por Jung (1964) y el trabajo experiencial de terapeutas gestálticos, analizaron cientos de sesiones de terapia en las cuales ocurrían estos cambios revolucionarios. El fruto de su esfuerzo fue la creación de un procedimiento terapéutico al que llamaron terapia breve de orientación profunda (Ecker y Hulley, 1996) por su capacidad de promover la reconstrucción del material inconsciente (estos autores se refieren a memoria inconsciente o implícita -opuesta a la memoria consciente o explícita- como el tipo de memoria que almacena, entre otros, habilidades motoras y linguistícas, hábitos y respuestas conductuales provocadas por las emociones; y cuya expresión no incluye atención consciente, de acuerdo con Toomey y Ecker, 2007) dentro de un formato de terapia de corta duración. Este enfoque, conocido actualmente como terapia de la coherencia, con el tiempo ha sido respaldado por hallazgos de la neurociencia, que a inicios del siglo XXI demostraron que es posible la reconsolidación de las memorias implícitas. El proceso llevado a cabo por la terapia de la coherencia es un reflejo de los pasos requeridos por el cerebro para que se produzca este cambio en la memoria.
La terapia de la coherencia pertenece a la familia de los enfoques constructivistas (Feixas y Villegas, 2000; Neimeyer y Mahoney, 1995) con los que comparte la visión del ser humano como agente activo que construye la realidad experiencial en la que opera y vive de una forma que es personal y, en último término, única. De esta forma, evitando la clasificación o patologización de las conductas, se centra en cómo el individuo subjetivamente construye su realidad no solamente de forma explícita sino, muy especialmente, con sus construcciones implícitas.

Concebida como un enfoque experiencial de corta duración la terapia de la coherencia puede ser aplicada en el trabajo con individuos, parejas y familias. Como principio central propone que el síntoma es coherente con significados implícitos, raramente conscientes, que surgen de construcciones de la realidad que fueron adaptativas en el pasado y que actualmente siguen activas, dando lugar directa o indirectamente al síntoma. Uno de los aspectos más distintivos de los enfoques constructivistas en general, y de la terapia de la coherencia en particular, es que el terapeuta no se opone al síntoma, en lugar de esto, guía al cliente a descubrir de forma experiencial los esquemas que le dan sentido y promueve su integración a nivel consciente. Es así que estas construcciones son modificadas, haciendo que el síntoma pierda su significado original. De acuerdo con los autores de este enfoque (Ecker et al., 2012; Ecker y Toomey, 2008), los pasos que conducen al cambio terapéuticamente efectivo son un reflejo del proceso de reconsolidación de la memoria, un proceso neuronal que permite que se produzca un cambio duradero, a nivel de los tejidos cerebrales, en las conexiones de la memoria implícita.

\section{Constructivismo y psicoterapia}

Antes de describir la terapia de la coherencia parece conveniente explicar brevemente la influencia del pensamiento constructivista sobre la psicoterapia y la ciencia en general.

Las psicoterapias constructivistas (véase, por ejemplo, Feixas y Villegas, 2000) prestan atención a los significados que el individuo otorga a la realidad que experimenta 
y a como la construcción subjetiva de la misma da forma a su propia experiencia. El pensamiento denominado constructivista aparece en la psicología en los años setenta (Watzlawick, 1976), dos décadas después que George Kelly publicará su opus magnum, La psicología de los constructos personales, en 1955. Tanto Kelly como los constructivistas que lo sucedieron no formularon sus teorías a partir de la nada. Ya en el siglo XVII, Giambattista Vico e Immanuel Kant, y en el siglo XX Hans Vaihinger y Jean Piaget enfatizaban el papel activo de la mente en la construcción de la realidad (Mahoney, 1988). Sin embargo, no es hasta los años setenta que el constructivismo surge como un nuevo enfoque a partir de la evidencia empírica recolectada en diferentes campos científicos, en especial en la neuropsicología, la biología y la cibernética. Fueron los representantes de las ciencias naturales como Heinz von Foerster (1981), Humberto Maturana y Francisco Varela (1987); de la filosofía como Ernst von Glasersfeld (1984), y de la psicoterapia como Paul Watzlawick (1976), quienes formularon las nociones básicas del constructivismo. Actualmente estas premisas coinciden con hallazgos científicos que plantean a la cognición como un proceso activo y generativo que depende más de la estructura del cerebro y sus reglas de funcionamiento que del mundo exterior (Neimeyer, 2009). Tal conocimiento permitió a von Glasersfeld (1989, p. 162) formular sus principios constructivistas:

1) "El conocimiento no se recibe de forma pasiva a través de los sentidos o por la comunicación, sino que activamente construido por el sujeto que lo percibe.

2) La cognición sirve para la organización del mundo experiencial del sujeto, no para el descubrimiento de una realidad ontológica objetiva". (p.162).

Estas afirmaciones nos invitan a dejar a un lado la búsqueda racionalista por descubrir una verdad independiente del observador. No obstante, la existencia de la realidad, a nivel ontológico, no es negada por los constructivistas; sin embargo, si no es posible conocerla objetivamente, entonces la verificación del conocimiento construido mientras se experimenta el mundo exterior ocurre recursivamente con la acumulación de nuevas experiencias -es a partir de esto que surge la función adaptativa de la cognición (Neimeyer, 2009). Tanto Kelly como los científicos mencionados anteriormente percibieron que el ser humano formula ciertas hipótesis las cuales son verificadas en la práctica y corregidas de acuerdo a los criterios de adaptación- las hipótesis confirmadas refuerzan la construcción otorgada a la realidad mientras que las que se invalidan demandan ser modificadas (Ke1ly, 1955/1991). Von Foerster enfatiza que la base para detectar la diferencia no es dada por el mundo exterior, sino por nuestras propias construcciones en relación al mundo (Foerster, 1973).

Los enfoques psicoterapéuticos que se basan en el constructivismo han abandonado la fe en una única realidad correcta y han tomado una visión escéptica sobre las definiciones objetivistas acerca de la salud mental y los trastornos. El terapeuta ha dejado de ser un experto directivo que identifica las estructuras a cambiar por parte del cliente (Kelly, 1955/1991). Kelly mencionó que nadie tiene que ser prisionero de su propia biografía y que todo lo que es construido mentalmente puede ser reconstruido (Kelly, 1969). Mientas que las terapias objetivistas tradicionales se centran en la corrección de acciones o regulación emocional, los terapeutas constructivistas, en lugar de luchar contra el síntoma, ayudan a los clientes a expandir su sistema de constructos personales (o narrativas personales) de tal manera que el síntoma pierda las razones para existir. Así pues, la psicoeducación y la eliminación de los patrones disfuncionales son remplazados por la exploración y reconstrucción de significados. En cuanto a las emociones negativas, estas no son un signo de patrones de pensamiento distorsionados, sino que informan de la existencia de un conflicto entre las construcciones existentes. La resistencia es vista como una fuente de información acerca de que el cambio propuesto amenaza a las construcciones centrales que son vitales para mantener la identidad del cliente (Kelly, 1969).

Las terapias constructivistas contemporáneas comparten la visión de que la realidad objetiva es imposible de llegar a conocer, es el ser humano quien activamente y de forma adaptativa organiza su construcción subjetiva de la realidad (Neimeyer, 2009). Teniendo en cuenta que la característica común de estos enfoques es la epistemología y no necesariamente las teorías o las estrategias específicas, dentro de la familia de las terapias 
constructivistas es posible encontrar tanto terapias cognitivas (por ejemplo, adaptaciones modernas de la psicoterapia de los constructos personales o la terapia postracionalista de Guidano), como terapias experienciales (la terapia focalizada en emociones de Greenberg o la terapia de la coherencia). El constructivismo también ha influenciado el desarrollo de las terapias estratégicas (atribuidas al pensamiento de Bateson y Watzlawick) y en conjunto con el construccionismo social han influido sobre el desarrollo de las terapias sistémicas. Además, existen modelos constructivistas y socioconstruccionistas desarrollados para el tratamiento de problemas específicos; por ejemplo, la terapia del duelo de Neimeyer (Neimeyer, Burke, Mackay y Van Dyke Stringer, 2010) el enfoque de Semerari y Dimaggio sobre los trastornos de personalidad (2011) o el enfoque del diálogo abierto para el tratamiento de la psicosis de Seikkula (Seikkula, Alakare, Aaltonen, 2001).

En la descripción presentada del constructivismo, el lector podrá identificar algunas similitudes con el construccionismo social. Las analogías que rigen estas nociones han sido confundidas entre ellas o usadas de manera intercambiada. El construccionismo social es un paradigma similar, en ocasiones percibido como resultado del desarrollo del pensamiento constructivista que pone énfasis sobre la construcción de la realidad dentro del espacio que hay entre individuos (en un amplio contexto social y cultural, con el uso del lenguaje), en lugar de la construcción individual (Kelly, 1955/1991). En psicoterapia, esta corriente ha contribuido a poner en el foco de atención a los determinantes políticos, sociales y lingüísticos que permitieron la producción de cambios vitales en las terapias sistémicas y contribuyeron a la aparición de la terapia narrativa (White y Epston, 1990).

\section{Orígenes de la terapia de la coherencia}

Bruce Ecker y Laurel Hulley, una pareja de psicoterapeutas de California, formados inicialmente en el enfoque sistémico, notaron que en su práctica clínica las sesiones de terapia más efectivas eran aquellas en las cuales, contrario a las pautas de las terapias sistémicas, trabajaban sobre las construcciones inconscientes de la realidad de los clientes. Sin embargo, realizaban este trabajo desde la perspectiva constructivista, no desde la perspectiva psicodinámica. Observaron que los clientes de manera especialmente rápida lograban el cambio deseado cuando de manera experiencial (no únicamente cognitiva o desarrollada por la interpretación del terapeuta) descubrían el significado inconsciente del problema, integraban este conocimiento a nivel consciente y entonces lo reconstruían de tal forma que el síntoma perdía sus razones para existir o dejaba de ser un problema (Ecker y Hulley, 1996). Así pues, estos terapuetas comenzaron a crear un sistema terapéutico que pudiera, desde su punto de vista, facilitar la realización de tales sesiones efectivas con mayor regularidad. En un inicio el desarrollo de este enfoque fue denominado por Ecker y Hulley como terapia breve de orientación profunda, que salió a la luz en 1996 con la presentación del libro con el mismo nombre (en inglés Depth-Oriented Brief Therapy). Nueve años más tarde los autores cambiaron el nombre del enfoque por terapia de la coherencia con el objetivo de enfatizar su principio básico-la coherencia del síntoma con las construcciones adaptativas que requieren su mantenimiento. Aquel periodo dio también un importante impulso para el desarrollo del pensamiento de Ecker y Hulley, incluso Ecker (Ecker y Toomey, 2007) se preguntó a sí mismo sobre la existencia de una explicación neurológica para la alta efectividad de la terapia de la coherencia. No obstante, tal explicación comenzó a existir relativamente desde hace pocos años.

\section{Coherencia entre psicoterapia y neurociencia}

En la descripción presentada del constructivismo, el lecto El desarrollo de la neurociencia en las dos últimas décadas ha reforzado los fundamentos científicos de la psicoterapia, los cuales en ocasiones han sido criticados por ser insuficientes e incluso por la falta de los mismos. La investigación sobre la reconsolidación de la memoria, retomada en 1997, apareció como un elemento revolucionario que parte de la idea de confirmar que tal proceso es posible. En el 2004 el proceso requerido para que 
exista la reconsolidación fue definido y este se convirtió en el segundo pilar, después de la práctica clínica, para la terapia de la coherencia. Ecker sostiene que el análisis de las transcripciones de las sesiones de terapia permite identificar los marcadores del cambio profundo igual a los que se observan en el proceso de reconsolidación (Ecker et al., 2012).

La memoria inconsciente (llamada también "implícita") es un tipo de memoria a largo plazo. Su contenido es recuperado y nos influye automáticamente sin la necesidad de involucrar conexiones del nivel consciente. Las conexiones (o engramas) de este tipo de memoria son en ocasiones creadas por la fuerte saturación emocional, como por ejemplo la presencia de traumas o eventos específicos vitales en la vida de un individuo. Los científicos ya en el siglo XX estaban interesados en las posibilidades de cambiar o borrar segmentos elegidos de memoria inconsciente, pero sus esfuerzos en conseguir la reconsolidación de la memoria fracasaron, así que se comenzó a considerar de manera enfática que este tipo de memorias se consolidan de una manera indeleble con el objetivo de protegerse, por lo tanto, es imposible cambiarlas (LeDoux, Romanski y Xagoraris, 1989). Esta fue una conclusión muy pesimista tanto para neurocientíficos, como para psicoterapeutas, ya que el conocimiento almacenado en la memoria implícita se considera, entre otros, que está relacionado con nuestra construcción de los principios básicos del funcionamiento del mundo $\mathrm{y}$, por consiguiente, con el saber de como uno tiene que comportarse para evitar el hacerse daño y obtener placer.

¿Y la psicoterapia cómo funciona en esta situación? Bien, si uno no puede influenciar en la memoria inconsciente, la solución está en crear nuevas conexiones que compitan efectivamente con las conexiones problemáticas existentes. Esta idea ha estado detrás de muchas escuelas terapéuticas que promueven la acción en contra del síntoma, por ejemplo, mediante la relajación, la psicoeducación, el pensamiento positivo, la regulación emocional, las reacciones alternativas, etc. A pesar de la buena fe en base de estas medidas, a menudo demandan un esfuerzo continuo del cliente para "silenciar" el síntoma (de seguro hemos escuchado más de una vez como alguien ha aprendido cómo "lidiar con la ansiedad"). Cierto es que Albert Ellis, el fundador la terapia racional emotiva, fue específico señalando el manejo de la memoria inconsciente, pero desde la visión de la neurociencia contemporánea, se refirió en exceso al trabajo de las emociones desde una perspectiva cognitiva. La fuente del problema se mantenía intacta-las conexiones de la memoria no deseada, consolidadas en el pasado, no estaban siendo cambiadas por las conexiones neuronales con los que competían, ya que estos estaban formados en la parte del cerebro más joven desde una perspectiva evolutiva (en el neocortex); mientras que la memoria emocional está codificada en el allocorteza, en el sistema límbico entre otros (Ecker et al., 2012).

La investigación sobre la reconsolidación de la memoria fue retomada en 1997. Tres años después Nader y sus colaboradores confirmaron la hipótesis que proclamaba que las conexiones neuronales de las memorias inconscientes consolidadas relacionadas con el miedo pueden ser desestabilizadas (regresar a un estado no consolidado, lábil en el que pueden ser modificadas, reforzadas o borradas; Nader, Schafe y LeDoux, 2000). Este revolucionario hallazgo dio como resultado la intensificación de la investigación sobre este tema con el fin de precisar las afirmaciones e hipótesis planteadas. Muchos laboratorios comenzaron a experimentar en diferentes especies, incluso con humanos. El suceso de reconsolidación en humanos ha sido experimentado tanto con el uso de agentes farmacológicos que acentúan el proceso (específicamente el propanolol) como mediante el uso de intervenciones conductuales (Nader, 2013). Actualmente se considera que la reconsolidación de la memoria es posible únicamente cuando la memoria reactivada es contrapuesta con un conocimiento que la contradice, para ser más precisos, que contradice o es considerablemente diferente a las anticipaciones que establecía la memoria original. Únicamente con estas condiciones satisfechas este segmento de memoria se vuelve frágil y por tanto propenso al cambio (Oyarzún, et al., 2012; Schiller, Monfils, Raio, Johnson y LeDoux, 2010). Los investigadores fueron incluso capaces de definir el marco temporal en el cual la memoria implícita es modificable - la llamada ventana de la reconsolidación permanece abierta por alrededor de cinco horas a partir de la reactivación de la memoria y la contraposición del conocimiento mencionado anteriormente. Cuando termina este tiempo el engrama se vuelve a consolidar, lo cual hace imposible la introducción de cual- 
quier cambio (Phelps y Schiller, 2013). La verificación de la eficacia del proceso consiste en la reactivación del engrama de la memoria original con el fin de comprobar si este continúa causando una reacción emocional intensa-si este no es el caso, entonces se asume que la reconsolidación ha sido exitosa. Esto es valioso si mencionamos que la modificación de la memoria emocional elegida no causa el olvido del evento, ya que este proceso no actúa sobre la memoria autobiográfica, únicamente lo hace sobre los esquemas emocionales que fueron formados en el momento en el que el evento ocurrió (Ecker et al., 2012).

La reconsolidación de la memoria es considerada actualmente como el único mecanismo de la neuroplasticidad cerebral que permite el borrado o modificación permanente de un conocimiento inconsciente de una memoria emocional determinada. Desde el 2005, Ecker se ha dedicado a explicar el fenómeno de la efectividad de la terapia de la coherencia mediante el proceso de reconsolidación de la memoria e integrar la neurociencia y la psicoterapia con el objetivo de crear un marco teórico que permita el desempeño de una terapia efectiva independientemente de un modelo teórico concreto. En tres artículos publicados entre 2007 y 2009 en la revista de psicología constructivista (Journal of Constructivist Psychology) y en el libro Reconsolidación de la memoria: desbloqueo del cerebro emocional para la erradicación de los sintomas en psicoterapia (Unlocking the emotional brain: Eliminating Symptoms at Their Roots Using Memory Reconsolidation), en 2012, Ecker y sus colaboradores describen las implicaciones de los avances en neurociencia para la psicoterapia y argumentan que el constructivismo es el paradigma adecuado para introducir estos avances en la práctica clínica. La terapia de la coherencia se plantea como un método apropiado, aunque no el único que cumple con estos objetivos (Ecker et al., 2012; Toomey y Ecker, 2007). A continuación ofrecemos una mirada más cercana sobre este enfoque.

\section{Los supuestos de la terapia de la coherencia y su metodología}

La base de la terapia de Ecker y Hulley es el ya mencionado principio de la coherencia del síntoma. Este supuesto no es ni nuevo ni original, ya en el pasado fue resaltado por Jung, Watzlawick o Satir; y las posibles asociaciones con el concepto de funcionalidad del síntoma son legítimas (Toomey y Ecker, 2007). La contribución de los creadores de la terapia de la coherencia recae en la visión de esta idea de manera constructivista, no patologizante que se desarrolla alrededor de un sistema terapéutico claro, el cual según los autores hace posible el aumento de la efectividad de la psicoterapia. En otras palabras, toda la metodología de la terapia de la coherencia está construida alrededor del principio de la coherencia del síntoma. Ecker y Hulley (1996) ponen especial atención al hecho de que los seres humanos necesitan narrativas convincentes no solo a nivel consciente (enfatizado por muchas escuelas terapéuticas), pero también a nivel de construcciones inconscientes. El síntoma es percibido como negativo para la persona y los que le rodean; sin embargo, es compatible con al menos un esquema adaptativo de la realidad, codificado en la memoria implícita en un momento concreto de la vida. Si las anticipaciones que resultan de este esquema permitieron al individuo adaptarse mejor a su contexto en el pasado, estas pueden estar activas en el presente hasta el momento en que son descubiertas, integradas en el nivel consciente y reconstruidas. En otras palabras, tan pronto como dejen de existir construcciones de la realidad que requieran del síntoma, la persona dejará de producirlo (Ecker y Hulley, 2011).

Los creadores de la terapia de la coherencia manifiestan que llegar a conocer las construcciones inconscientes no debe tomar muchas sesiones, porque están bien definidas y continuamente activas, lo que significa que están disponibles y pueden ser transformadas desde el inicio del proceso terapéutico. Una condición que es necesaria para conseguir estos efectos es el trabajo experiencial, el cual tiene como objetivo descubrir el esquema que necesita de la presencia del síntoma, no mediante la contraposición 
que será la presunción inicial del cliente - un deseo natural de deshacerse del problema. Ecker y Hulley definen esta postura como la posición anti-síntoma. Como fue mencionado anteriormente, permanecer en esta posición no otorga ningún control sobre el síntoma y no permite una transformación duradera del material inconsciente lo que termina en frustración para el cliente (y en ocasiones para el terapeuta). La terapia de la coherencia sostiene que el cliente recobra el sentido de agencia cuando toma la posición pro-síntoma. Por lo tanto, el terapeuta evita cualquier práctica que pueda contraponerse al síntoma y guía al cliente hacia un descubrimiento experiencial de las construcciones inconscientes que lo mantienen (Ecker y Hulley, 1996). Esta guía adopta el enfoque de actitud crédula propuesta por Kelly (1955/1991), lo que significa estar abiertos y aceptar las construcciones subjetivas de la realidad del cliente y en consecuencia no imponer las interpretaciones propias del terapeuta. El trabajo experiencial excluye también el análisis del problema exclusivamente a nivel cognitivo -en lugar de hablar acerca del problema el terapeuta sugiere experimentar la realidad emocional relacionada con el mismo. Por consiguiente, un insight cognitivo no provoca el entendimiento del material inconsciente- experimentar este material es lo que promueve tal insight. El terapeuta conduce al cliente a través del proceso terapéutico y al mismo tiempo sigue de forma cautelosa los descubrimientos que hace la persona, sin añadir sus propias interpretaciones.

Ecker y Hulley (2011) ofrecen un conjunto de técnicas (algunas de ellas originales, otras ajustadas a las necesidades del proceso terapéutico) las cuales ayudan a conseguir los objetivos planteados anteriormente. Al mismo tiempo enfatizan la necesidad de acercarse a las necesidades individuales de cada cliente y la libertad para que el terapeuta elija los métodos siempre y cuando sean experienciales y cumplan con los supuestos de la TC. Los autores definen de manera muy concreta cuáles son los pasos intermedios y los objetivos que el terapeuta debe alcanzar con el fin de establecer que la terapia sea efectiva, pero la forma de conseguirlos (en su mayoría) depende del terapeuta -Ecker utiliza la metáfora del mapa- conocemos el punto final, pero somos libres para escoger la manera de llegar allí (Ecker y Hulley, 2011).

Demos un vistazo de lo que es la posición prosíntoma (llamada también la verdad emocional del síntoma) la cual consiste en dos elementos: el primero es la memoria emocional, somática o perceptual de la experiencia original -puede ser por ejemplo una sensación de aislamiento o rechazo que se produce cada vez que la persona es injustamente evaluada por su padre por un error cometido en la niñez. El segundo elemento es el modelo del problema y su solución. El problema en este caso es el peligro a ser criticado o rechazado si se comente el más mínimo error. La solución recae sobre un amplio rango de conductas que pueden ser contrapuestas en muchos enfoques terapéuticos desde la posición anti-síntoma: el perfeccionismo, falta de asertividad, incapacidad de tomar decisiones, procrastinación, ansiedad paralizante, etc. Sin embargo, los creadores de la TC recalcan que tales soluciones son adaptativas y sirven para evitar un sufrimiento incluso peor que el causado por el síntoma (Ecker et al., 2012). Es válido mencionar aquí que el síntoma en ocasiones no tiene la función de una solución, pero es derivado de construir un esquema adaptativo determinado. Este puede ser el caso por ejemplo de un aislamiento proveniente de la ansiedad que que por sí misma puede ser adaptativa (Ecker y Hulley, 2011).

En conclusión, la terapia de la coherencia, similar a los enfoques psicodinámicos subraya la importancia de las construcciones inconscientes. Sin embargo, estas no son vistas a través de planteamientos teóricos patologizantes y la herramienta que permite su descubrimiento no es la interpretación, definitivamente evitada en el enfoque descrito. El descubrimiento de la posición pro-síntoma no ocurre por medio del análisis cognitivo o psicoeducativo que pondría al terapeuta en el rol de "experto" como lo hace la terapia cognitivo conductual. Los síntomas no son tratados como resultantes de errores cognitivos que deben ser corregidos debido a su irracionalidad o disfuncionalidad, sino como elecciones personales (inconscientes, pero adaptativas). Las acciones correctoras son evitadas ya que la contraposición del síntoma, típica de las terapias cog- 
nitivas tradicionales, es contradictoria al principio de la coherencia del síntoma y, según Ecker y colaboradores (2012), no elimina el síntoma. La posición directiva del terapeuta sólo se considera a través de ser un guía en el proceso de experimentar, integrar y cambiar las construcciones subjetivas de la realidad, las cuales no han sido manifestadas a nivel consciente anteriormente. De este modo, similar a otras terapias constructivistas, el terapeuta y el cliente se fijan juntos en la reconstrucción de la realidad idiosincrática que permitirá la resolución de los conflictos existentes sin amenazar a las construcciones que son importantes para la identidad del cliente. La terapia de la coherencia, como otros enfoques experienciales contemporáneos (por ejemplo, la terapia centrada en las emociones de Greenberg), asume que el cambio terapéutico, para ser duradero, tiene que estar anclado a la experiencia emocional. Esta postura es compartida también por los constructivistas contemporáneos (Neimeyer, 1993) y la efectividad de la mayoría de los métodos experienciales usados en el enfoque de Ecker y Hulley han sido confirmados empíricamente (Elliott et al., 2003).

\section{El proceso terapéutico}

Como se ha mencionado, la terapia de la coherencia pretende guiar a los clientes a través de etapas concretas que hacen posible el proceso de reconsolidación de la memoria. Ecker et al. (2012) trasladan el proceso explicado por la neurociencia al ambiente clínico y presentan un marco metodológico que puede ser, según ellos, identificado no solamente en la terapia de la coherencia, sino también en otros enfoques experienciales, por ejemplo en la terapia centrada en las emociones, desensibilización y reprocesamiento por los movimientos oculares (eye movement desensitization and reprocessing - EMDR) o psicoterapia dinámica experiencial accelerada (accelerated experiential dynamic psychotherapy AEDP). Los autores de Unlocking the Emotional Brain notaron que las escuelas mencionadas promueven la reconsolidación de la memoria a pesar de diferenciarse de la terapia de la coherencia. Partiendo de este hecho, es posible afirmar que este procedimiento puede ser conducido efectivamente por otros enfoques, siempre y cuando el terapeuta trate de llevarlo a cabo de manera consciente y competente. Con esto, los fundadores de la terapia de la coherencia muestran un posible camino para la integración en psicoterapia que se basa en el proceso que necesita el cerebro para conseguir un cambio duradero, en lugar de fundamentarse en supuestos teóricos y técnicos (Ecker et al., 2012).

Ecker afirma que el proceso universal de reconsolidación en terapia debería consistir en tres etapas (descubrimiento con integración de las construcciones del inconsciente, sus transformaciones y verificación del cambio), divididas en pasos específicos, definidos como A, B, C, 1, 2, 3, V (Ecker, et al., 2012). Abordemos este proceso mediante el análisis de un estudio de caso simplificado de un hombre de 33 años llamado Jorge que acude a terapia quejándose de unos síntomas elevados de hipocondría acompañados de ansiedad y un estado de ánimo depresivo. Los síntomas aparecieron por primera vez hace unos meses y últimamente han llegado a un nivel insoportable. Jorge cuenta que en el pasado nunca había sufrido de hipocondría, aunque admite que siempre ha sido una persona bastante preocupada y durante algunos años tenía miedo a volar en avión, pero pudo superar este problema. El terapeuta se muestra empático y valida el deseo de Jorge de dejar a un lado el síntoma, pero desde el inicio intenta guiarle a descubrir la posición prosíntoma. El primer paso (paso A) es definir el síntomaa partir de indagar cuándo y cómo aparece el problema y cuál es el significado que se le ha otorgado. Para poder hacer esto, el terapeuta pide al paciente que vuelva a una situación reciente en la que la hipocondría lo molestó especialmente y que la describa detalladamente (a lo mejor con ojos cerrados) como si estuviera ocurriendo en el presente, incluyendo las sensaciones fisiológicas, emociones y pensamientos que aparecen en su mente al ir relatando tal situación. El uso de técnicas experienciales (en lugar de analizar el problema a nivel cognitivo) concede al terapeuta información acerca de los pensamientos y emociones específicas del cliente. Jorge dice que en tales situaciones se siente desesperado y muy preocupado por su estado de salud. Se fija totalmente en el problema 
actual que le preocupa y le produce la ansiedad (por ejemplo, la posibilidad de tener un cancer de estómago), así intenta resolverlo buscando un diagnóstico a través de visitas a varios médicos y búsqueda de información en libros e Internet. Después de conseguir una descripción detallada del síntoma, podemos pasar al siguiente paso.

En el paso B el interés del terapeuta se centra en la posición pro-síntoma. El terapeuta tiene que guiar al cliente a contestar la pregunta acerca de cuáles son las construcciones inconscientes que hacen necesario el síntoma. Una de las opciones sugeridas por Ecker y Hulley (1996) para poder alcanzar este objetivo es usar la técnica de deprivación del síntoma. Esta consiste en guiar al cliente a través de una situación en la que el síntoma haya aparecido (con todos los detalles posibles), quitando de esta situación el síntoma, es decir experimentar la situación sin el síntoma. En el ejercicio terapéutico, el cliente experimenta exactamente la misma secuencia como si estuviera pasando en el presente, pero en el momento en el que en la situación original apareció el sentimiento de ansiedad y preocupación relacionada con su salud se le invita a experimentar como le hubiese gustado sentirse, por ejemplo relajado y animado. El cliente tiene que sentir este estado de forma auténtica, por eso se pone mucho énfasis en guiarlo a través de la situación de una manera vivencial. Esta técnica está basada en el postulado de que si el síntoma es necesario, su eliminación activará sentimientos negativos específicos (resultado de la posición pro-síntoma) que son evitados gracias a la presencia del síntoma. Cuando el cliente empieza a sentir las emociones negativas, el terapeuta lo guía hacía la verbalización de lo descubierto. Jorge, al ser deprivado de su síntoma, siente que no debería estar relajado y gozar de una vida sin ansiedad, que algo no está bien en vivir así. El terapeuta le ayuda a verbalizar el material descubierto hasta este momento y sugiere apuntarlo en una frase que se construye conjuntamente: "Si estoy relajado y gozo de una vida sin ansiedad, algo no está bien. Sé que no debería vivir de esta manera". El terapeuta apunta esta frase en una tarjeta y la entrega a Jorge para que este la lea en voz alta y conecte con las emociones que surgen de esta actividad. El terapeuta le pide que cierre los ojos y se quede con estas sensaciones para descubrir la razón profunda de no poder estár relajado y tranquilo. Gracias a profundizar esta experiencia es posible reconocer constructos de orden superior que definen las reglas de organización de la realidad. Así Jorge llega a descubrir algo de lo que no ha sido consciente hasta ahora: si está relajado y disfruta la vida, seguro que pasará algo que lo estropeará. La frase apuntada en la segunda tarjeta explica esta verdad emocional de una manera muy clara: "Si estoy relajado y vivo tranquilo, seguro pasará algo horrible - alguna catástrofe que lo destrozará todo, pues mejor estar preparado y así evitarlo-preocuparse en vez de mentirse a uno mismo pensando que la vida es fácil". De esta manera el cliente descubre que la hipocondría y la ansiedad en general son sus maneras para evitar esta catástrofe tan temida. Al buscar los orígenes de esta construcción adaptativa, se llega a conocer que los padres del cliente son personas que siempre se han preocupado excesivamente por cualquier aspecto de la vida familiar. Especialmente el padre, al parecer, le inculcó al cliente que uno siempre tiene que pensar en el futuro, prepararse para lo peor y nunca "bajar la guardia". En esta familia el mundo exterior es entendido como algo hostil, lleno de peligro y desgracias que le pueden pasar a uno si no está atento esta es la definición del problema a partir de la posición pro-síntoma del cliente. La solución es estar ansiososiempre preocupado y preparado para lo peor, también en cuanto a la salud (de aquí viene la hipocondría). El descubrimiento de este esquema explica la imposibilidad de vivir en concordancia con la posición anti-síntoma (ser una persona tranquila, relajada). Por lo tanto, el cliente permanece en el estado actual, indeseado pero adaptativo desde su perspectiva. La construcción descubierta tiene que ser integrada a nivel consciente, para lograrlo se usan técnicas que permiten que ambas posiciones sean experimentadas en el mismo nivel de atención, tanto en la sesión como fuera de ella.

En el paso $\mathrm{C}$ se necesita identificar el conocimiento que pueda contradecir la construcción descubierta. En el caso de Jorge encontramos varios hechos de su vida que se oponen a esta construcción tan bien arraigada. $\mathrm{Su}$ hermana no es una persona ansiosa y nunca le ha pasado 
nada malo, es más-disfruta de su vida y parece ser feliz. Lo mismo puede decir Jorge de algunos amigos cercanos. A su padre, en cambio, no le ha ayudado mucho esta actitud porque por estar siempre preocupado no ha tenido una vida muy interesante. Además, su seguridad al fin y al cabo parece algo frágil-Jorge llega a la conclusión que aunque uno se preocupe mucho, nunca podrá estar $100 \%$ seguro de que no le pasará nada malo. De esta manera se ha descubierto el conocimiento que podría contradecir a las construcciones que gobiernan la realidad subjetiva del cliente.

La siguiente etapa es crucial para la terapia de la cohrencia. En la fase de transformación se produce el proceso de la reconsolidación de la memoria. En el paso 1 se reactiva el esquema que tenemos como objetivo-se pide al cliente que vuelva de manera experiencial a la situación en la cual usualmente toma la posición pro-síntoma, o sea se pone ansioso y/o hipocondríaco. Seguidamente (paso 2) el terapeuta, en el mismo campo de atención, de una manera completamente imparcial, pide al cliente que imagine el nuevo conocimiento que es contradictorio-que se imagine a su hermana que es una persona relajada y no le pasa nada, que mire a sus amigos y al padre que no es feliz siguiendo la pauta pro-síntoma. Esto conduce a que el engrama de la memoria donde se ha codificado la construcción en cuestión se vuelva lábil y la ventana de reconsolidación se abra. Ecker denominó experiencia de yuxtaposición a la experiencia simultánea del conocimiento cimentado en el inconsciente, las construcciones descubiertas y el conocimiento que las contradice directamente o que es muy distinto de ellas. Esta experiencia se basa en el supuesto de que el cerebro permite que existan dos construcciones contradictorias siempre y cuando ellas no sean experimentadas en el mismo campo de atención y al mismo tiempo. Cuando esto pasa, el cerebro tiene que hacer una elección porque estas construcciones no pueden ser ambas verdaderas ya que se contradicen entre ellas al mismo tiempo. La construcción original es cambiada o actualizada por la nueva que reconstruye la realidad del cliente, como resultado cesan los síntomas (Ecker y Toomey, 2008). A fin de que esto ocurra, el terapeuta debe repetir la experiencia de yuxtaposición algunas veces. Para esto (paso 3) se guía al cliente de manera alternada a través del conocimiento original (Jorge debe imaginarse de nuevo la posición prosíntoma y lo que esta significa para él) y el nuevo conocimiento (imaginarse otra vez todas o algunas de las contradicciones encontradas) debido a esta "disonancia experiencial" específica, el cambio esperado puede ocurrir (Ecker et al., 2012).

De este modo, Jorge (o más bien su mente inconsciente) elige la nueva "verdad", que en este momento vital es más adaptativa. La última fase del proceso es la etapa de verificación (paso V) descrita anteriormente (el terapeuta activa nuevamente el esquema diana y observa la reacción del cliente). En el caso de Jorge el nuevo nivel de conciencia permite la aparición de la tristeza por haber vivido tantos años guiado por una visión del mundo que no le dejaba gozar de su vida, lo cual tiene que ser trabajado, pero ahora ya nada le impide comenzar a sentirse bien consigo mismo y disfrutar plenamente del presente, sin perder el lado bueno que tiene el ser responsable y organizado. Ahora, después de la reconstrucción de la realidad emocional, la hipocondría ya no tiene una función para él.

\section{Estado actual y el futuro de la terapia de la coherencia}

La terapia de la coherencia es bastante popular en Norte América donde ha sido incluida en los programas de entrenamiento de la Asociación Americana de Psicología (APA). El interés también ha aumentado en Europa y Australia. En cuanto a las confirmaciones empíricas de su efectividad, existe hasta ahora únicamente un ensayo controlado aleatorio. La eficacia de la terapia de la coherencia para la procrastinación y el perfeccionismo $(n=18)$ fue evaluada en comparación a una intervención estructurada de biblioterapia $(n=19)$. Los resultados indicaron que el grupo que recibió TC superó al grupo de biblioterapia en cuanto a la reducción del perfeccionismo (aunque con tamaño efecto modesto), sin embargo, estas diferencias no se 
evidenciaron con respecto a las medidas de procrastinación (Rice, Neimeyer y Taylor, 2011). Si bien esta investigación mostró resultados positivos en cuanto a la reducción del perfeccionismo, hay que considerar sus limitaciones (muestra pequeña y el tipo de grupo control). La falta de otros «ensayos controlados aleatorizados» no han permitido valorar la eficacia de la TC.

El mismo Ecker admite que se necesita más investigación para confirmar la eficacia de su enfoque. Sin embargo, es partidario más bien de la investigación cualitativa que de la cuantitativa; ya que la última, según él, especialmente en ensayos controlados aleatorios, no presta suficiente atención a los casos exitosos donde el factor de cambio específico de una experiencia emocional correctiva tiene un papel primordial para la efectividad de la terapia (Ecker et al., 2012; Ecker, 2013). Con este postulado, Ecker y sus colaboradores sugieren prestar más atención a la definición de factores específicos en vez de satisfacerse con lo definido hasta ahora dentro de la teoría de los factores no específicos. Ellos creen que el proceso terapéutico de la reconsolidación de la memoria es el factor crucial para la explicación de la alta efectividad de la terapia de la coherencia e indican la importancia de seguir investigando este proceso y su ocurrencia en la consulta terapéutica (Ecker, 2013). Por otro lado, el uso de fMRI antes, durante y después del proceso de la terapia de la coherencia y otro enfoque elegido que esté basado en la contraposición del síntoma, posiblemente permitiría a los investigadores definir las diferencias entre ellas a nivel del sistema nervioso y confirmar la hipótesis acerca de que el enfoque de los psicólogos californianos promueve la reconsolidación de la memoria (Ecker y Toomey, 2008).

La terapia de la coherencia, desde una vision constructivista de entender la psicoterapia (Ecker y Hulley, 1996; Jasiński y Feixas, 2015; Toomey y Ecker, 2007), sin duda integra de una forma interesante lo que ha sido efectivo en la practica clínica, con lo confirmado por la neurociencia contemporánea. Por un lado la facilitación de una experiencia emocional, bloqueada anteriormente, junto con la reflexión consciente del significado que se ha descubierto a través de esta experiencia (Baikie y Wilhelm, 2005; Elliott et al., 2003; Greenberg, Warwar y Malcolm, 2008; Pennebaker, 1997). Mientras que, por otro lado, permite la posibilidad de cambiar, a través del proceso de reconsolidación, los engramas de memoria anteriormente consolidados (Schiller et al., 2010; Walker et al., 2003; Xue et al., 2012). Definitivamente mayor investigación en el tema ayudaría a promover este enfoque tan prometedor. Ecker y sus colaboradores enfatizan que la reconsolidación de la memoria ocurre, más o menos accidentalmente, en muchos enfoques terapéuticos. Sus contribuciones recaen en proponer un tipo de psicoterapia que puede cambiar la aleatoriedad en la regla. En sus escritos (Ecker et al., 2012), indican la efectividad de la terapia de la coherencia sobre un amplio espectro de problemas psicológicos, admitiendo al mismo tiempo que esta puede no ser una buena opción en casos graves de trastornos de personalidad, bipolaridad o trastornos psicóticos. Támpoco se recomienda su uso con individuos que presenten síntomas que tienen sus raíces no en construcciones inconscientes adaptativas, sino en irregularidades biológicas (como depresión resultante del hipertiroidismo) genéticas $u$ neuronales o en un daño orgánico (Ecker et al., 2012; Ecker y Toomey, 2008). Cuando se trata de clientes con síntomas acuciantes (por ejemplo, ataques de pánico frecuentes o niveles extremos de ansiedad) se podría necesitar algo de ayuda para reducir la intensidad del síntoma antes de involucrarse en un proceso de terapia de la coherencia. En ocasiones, los terapeutas que trabajan con este enfoque pueden encontrar dificultades al momento de enfrentar a los clientes con, el a menudo complicado, material inconsciente, especialmente si la persona que se presenta en su oficina prefiere focalizarse en las cosas positivas y olvidarse del problema. En tales casos es valioso explicar al cliente como funciona la terapia de la coherencia y entonces invitarlo a enfrentarse a las construcciones que se encuentran detrás del síntoma de forma vivencial. Muchas premisas indican que esta forma de actuar puede otorgar al cliente una satisfacción más duradera. 


\section{Referencias}

Baikie, K. A. y Wilhelm, K. (2005). Emotional and physical health benefits of expressive writing. Advances in Psychiatric Treatment, 11, 338-346. http://dx.doi.org/10.1192/apt.11.5.338

Bateson, G. (1951). Information and codification: A philosophical approach. En J. Ruesch y G. Bateson (Eds.), Communication: The social matrix of psychiatry (pp. 168-211). New York: Norton.

Bateson, G. (1972). Steps to an ecology of mind. New York: Ballantine.

Ecker, B. (2013). Nonspecific common factors theory meets memory reconsolidation: A game-changing encounter? The Neuropsychotherapist, 2, 134-137.

Ecker, B. y Hulley, L. (1996). Depth-Oriented brief therapy. San Francisco: Jossey-Bass.

Ecker, B. y Hulley, L. (2011). Coherence therapy manual and training guide. Oakland: Coherence Psychology Institute.

Ecker, B. y Toomey, B. (2008). Depotentiation of symptom-producing implicit memory in Coherence Therapy. Journal of Constructivist Psychology, 21(87), $87-150$ http://dx.doi.org/10.1080/10720530701853685

Ecker, B., Ticic, R. y Hulley, L. (2012). Unlocking the emotional brain. New York: Routledge.

Elliott R. K., Greenberg L. S. y Lietaer G. (2003). Research on experiential psychotherapies. En M. J. Lambert (Ed.), Bergin and Garfield's handbook of psychotherapy and behavior change (pp. 493-539). New York: Wiley.

Foerster H., von (1973). On constructing a reality. En F. E. Preiser (Ed.), Environmental design research (pp. 35-46). Stroudsbug: Dowden, Hutchinson, \& Ross.
Greenberg, L. S., Warwar, S. H. y Malcolm, W. M. (2008). Differential effects of emotion-focused therapy and psychoeducation in facilitating forgiveness and letting go of emotional injuries. Journal of Counseling Psychology, 55, 185-196. http://dx.doi.org/10.1037/0022-0167.55.2.185

Jasiński, M. y Feixas, G. (2015, julio). Coherence therapy and its place on the constructivist landscape. Comunicación presentada en el XXI Congreso Internacional de Psicología de los Constructos Personales. Hatfield: Universidad de Hertfordshire. Recuperado de http://prezi.com/mm8kjjwwbuyl/?utm_campaign=sh are\&utm_medium $=$ copy\& $\mathrm{rc}=\mathrm{ex} 0$ share.

Jung, C. G. (1964). Man and his symbols. Garden City, New York: Doubleday.

Kelly, G. A. (1955/1991). The Psychology of Personal Constructs. New York: Norton.

Kelly, G. A. (1969). Man's construction of his alternatives. En B. Maher (Ed.), Clinical psychology and personality: the selected papers of George Kelly (pp. 66-93). New York: Wiley.

LeDoux, J. E., Romanski, L. y Xagoraris, A. (1989). Indelibility of subcortical emotional memories. Journal of Cognitive Neuroscience, 1, 238-243. http://dx.doi.org/10.1162/jocn.1989.1.3.238

Mahoney, M. J. (1988). Constructive metatheory: I. Basic features and historical foundations. Journal of Personal Construct Psychology, 1, 1-35. http://dx.doi.org/10.1080/10720538808412762

Mahoney, M. J. (1991). Human change processes: The scientific foundations of psychotherapy. New York: Basic Books.

Maturana, H. y Varela, F (1987). The tree of knowledge. Boston: New Science Library. 
Nader, K. (2013). The discovery of memory reconsolidation. En C. M. Alberini (Ed.), Memory Reconsolidation. New York: Academic Press.

Nader, K., Schafe, G. E. y LeDoux, J. E. (2000). Fear memories require protein synthesis in the amygdala for reconsolidation after retrieval. Nature, 406, 722726. http://dx.doi.org/10.1038/35021052

Neimeyer R. A. (2009). Constructivist Psychotherapy. New York: Routledge.

Neimeyer, R. A., Burke, L, Mackay, M. y Stringer, J. (2010). Grief therapy and the reconstruction of meaning: From principles to practice. Journal of Contemporary Psychotherapy, 40, 73-83. http://dx.doi.org/10.1007/s10879-009-9135-3

Neimeyer, R. A. (1993). An appraisal of constructivist psychotherapies. Journal of Consulting and Clinical Psychology, 61(2), 221-234. http://dx.doi.org/10.1037//0022-006X.61.2.221

Oyarzún, J. P., Lopez-Barroso, D., Fuentemilla, L., Cucurell, D., Pedraza, C., Rodriguez-Fornells, A. y de Diego-Balaguer, R. (2012). Updating fearful memories with extinction training during reconsolidation: a human study using auditory aversive stimuli. PLoS ONE, 7, e38849. http://dx.doi.org/10.1371/journal.pone.0038849

Pennebaker, J. W. (1997). Opening Up: The Healing Power of Expressing Emotion. New York: Guilford Press.

Phelps, E. A. y Schiller, D. (2013). Reconsolidation in humans. En C. M. Alberini (Ed.), Memory Reconsolidation. New York: Academic Press.

Rice, K. G., Neimeyer, G. J. y Taylor, J. M. (2011). Efficacy of Coherence Therapy in the Treatment of Procrastination and Perfectionism. Counseling Outcome Research and Evaluation, 2(2), 126-136. http://dx.doi.org/10.1177/2150137811417975
Schiller, D., Monfils, M. H., Raio, C. M., Johnson, D. F., LeDoux, J. E. y Phelps, E. A. (2010). Preventing the return of fear in humans using reconsolidation update mechanisms. Nature, 463, 49-53. http://dx.doi.org/10.1038/nature08637

Seikkula, J., Alakare, B. y Aaltonen, J. (2001). Open dialogue in psychosis. An introduction and case illustration. Journal of Constructivist. Psychology, 14 , http://dx.doi.org/10.1080/10720530125965

Semerari, A. y Dimaggio, G. (2011). Los trastornos de la personalidad: modelos y tratamiento. Bilbao, España: Desclée De Brouwer.

Toomey, B. y Ecker, B. (2007). Of neurons and knowings: Constructivism, coherence psychology, and their neurodynamic substrates. Journal of Constructivist Psychology, 20(3), 201-245. http://dx.doi.org/10.1080/10720530701347860

Von Foerster. H. (1981). Observing systems. Seaside, CA: Intersystems Publications,

Von Glasersfeld, E. (1984). An introduction to radical constructivism. En P. Watzlawick (Ed.), The invented reality: How do we know what we believe we know? Contributions to constructivism (pp. 1740). New York: Norton.

Von Glasersfeld, E (1989). Constructivism in Education. En T. Husen y T. N. Postlethwaite (Eds.), International encyclopedia of education (Vol 1, Suppl. 2, pp. 162-163). Oxford: Pergamon Press.

Walker, M., P., Brakefield, T., Hobson, J. A. y Stickgold, R. (2003). Dissociable stages of human memory consolidation and reconsolidation. Nature, 425, 616620. http://dx.doi.org/10.1038/nature01930

Watzlawick, P. (1976). How real is real? Confusion, disinformation, communication. New York: Random House. 
White, M. y Epston, D. (1990). Narrative means to therapeutic ends. New York: Norton.

Xue, Y.-X., Luo, Y.-X., Wu, P., Shi, H.-S., Xue, L.-F., Chen, C., ... y Lu, L. (2012). A memory retrievalextinction procedure to prevent drug craving and relapse. Science, 336, 241-245.

http://dx.doi.org/10.1126/science. 1215070 